\title{
Alterações Funcionais em Pacientes com Câncer de Cabeça e Pescoço e a Atuação da Fisioterapia Nestas Disfunções: Estudo de Revisão
}

\section{Functional Changes in Patients with Head and Neck Cancer and the Role of Physiptherapy in These Dysfunctions: Review Study}

\author{
Walkiria Shimoya-Bittencourt ${ }^{\text {ab*; }}$; Alanna Emanuelle da Silva ${ }^{\text {a }}$; Denyse Dourado Alencar ${ }^{\mathrm{a}}$; Tais Regina Alves de Arruda \\ Cristhiane Almeida Leite ${ }^{\mathrm{b}}$; Marcos Adriano Salício ${ }^{\mathrm{ac}}$ \\ ${ }^{a}$ Centro Universitário de Várzea Grande, Cursos de Fisioterapia, MT, Brasil \\ bUniversidade de Cuiabá, Cursos de Fisioterapia, MT, Brasil \\ 'Universidade Federal de Mato Grosso, Programa de Pós-Graduação Stricto Sensu em Epidemiologia, MT, Brasil \\ *E-mail: wshimoya@yahoo.com.br \\ Recebido: 05 de outubro de 2015; Aceito: 18 de janeiro de 2015
}

\begin{abstract}
Resumo
O número de casos de câncer, incluindo o de cabeça e pescoço, tem aumentado de maneira considerável em todo o mundo, tornando-se um dos mais importantes problemas de saúde pública mundial. As consequências desse tipo de neoplasia podem afetar profundamente a qualidade de vida das pessoas acometidas, pois o tratamento é agressivo, podendo levar a complicações pós-operatórias e a disfunções musculoesqueléticas que interferem nas atividades da vida diária, especialmente no que se refere à funcionalidade e à autoimagem. O estudo teve como objetivo conhecer as alterações funcionais em pacientes com câncer de cabeça e pescoço e a atuação da fisioterapia frente a essas disfunções. Foi realizado um estudo de revisão bibliográfica, utilizando as bases de dados eletrônicas Medline, Lilacs, e as bibliotecas virtuais Bireme e SciELO. Foram incluídos estudos nos idiomas inglês, português e espanhol, sem restrição ao período de publicação. Dentre as complicações pós-operatórias que levam a disfunções musculoesqueléticas, encontradas na literatura as comumente relatadas foram a dor e inabilidade funcional no ombro, limitação na amplitude de movimento, alteração e/ou perda de força na musculatura do membro superior e pescoço homolateral à cirurgia, entre outras. A fisioterapia se mostrou importante nos cuidados desses pacientes, de modo que deve ser realizada para melhorar o déficit funcional.
\end{abstract}

Palavras-chave: Neoplasias de Cabeça e Pescoço. Complicações Pós-Operatórias. Fisioterapia.

\begin{abstract}
The number of cases of cancers, including head and neck, has increased worldwide, becoming one of the most important problems of global public health. The consequences of this type of cancer can affect the quality of life of patients, once the treatment is aggressive and can lead to postoperative complications and musculoskeletal disorders that interfere with activities of daily life, especially when it comes to functionality and self-image. This study aimed to investigate the functional changes in patients with head and neck cancer and the role of physiotherapy to such impairment. A bibliographic review using the electronic databases Medline, Lilacs, and virtual libraries Bireme and SciELO was carried out, including studies in English, Portuguese and Spanish, without period restrictions. Among the postoperative complications that lead to musculoskeletal disorders, pain and functional disability in the shoulder, limited range of motion, change and / or loss of strength in the upper limb and ipsilateral neck muscles to the surgery were the most reported, among others. Physical therapy proved to be important in the care of these patients to improve the functional deficit.
\end{abstract}

Keywords: Head and Neck Neoplasms. Postoperative Complications. Physical Therapy Specialty.

\section{Introdução}

A expressão "tumores de cabeça e pescoço" compreende um grande número de neoplasias com diferentes características histológicas, resultantes de diferentes locais anatômicos, como a cavidade oral (lábios, língua, assoalho bucal e palato duro), faringe (orofaringe, a hipofaringe e a nasofaringe) e laringe, além da porção cervical do esôfago, seios paranasais, glândulas salivares, tireoide, paratireoide e pele ${ }^{1}$. O número de casos de câncer tem aumentado de maneira considerável em todo o mundo, principalmente a partir do século passado, configurando-se, na atualidade, como um dos mais importantes problemas de saúde pública mundial ${ }^{2}$.

Dentre todos os cânceres que incidem na região de cabeça e pescoço, $40 \%$ ocorrem na cavidade oral, $15 \%$ na faringe, $25 \%$ na laringe e o restante nos demais sítios remanescentes (glândulas salivares, tireóide) $)^{3}$.

Dados do INCA demonstraram que o câncer bucal ocupa o quinto lugar de incidência entre todos os tipos de câncer nos homens e o sétimo entre as mulheres, no Brasil, num total de 14.120 novos casos ${ }^{4}$.

No mundo, aproximadamente 200 mil casos novos de câncer de cabeça e pescoço são diagnosticados por ano. Além disso, o perfil da população com maior suscetibilidade ao carcinoma de células escamosas, o tipo mais frequente de câncer de boca, corresponde a indivíduos com idade superior a 50 anos, do sexo masculino, com baixa renda, analfabetos ou com baixa escolaridade e residentes em zonas rurais. Uma vez que esse tipo de tumor tem comportamento agressivo, com metástase cervical precoce, um dos fatores determinantes de melhor prognostico é a atuação rápida dos profissionais no 
diagnóstico precoce, encaminhamento e tratamento ${ }^{5,6}$.

A incidência de metástases regionais em carcinomas primários da boca e orofaringe pode chegar a $85 \%$. Nestes casos, o esvaziamento cervical com finalidade terapêutica para os casos com linfonodos clinicamente metastáticos é sempre indicado. Do mesmo modo, o tratamento eletivo das áreas cervicais é indicado em lesões da orofaringe, uma vez que a taxa de doença metastática oculta é alta, principalmente em lesões das lojas amigdalianas e da base da língua. Na maioria das vezes o esvaziamento cervical utilizado para os casos com linfonodos metastáticos consiste em uma das modalidades de esvaziamento radical ${ }^{7}$.

Além disso, os gânglios linfáticos mais frequentemente envolvidos estão localizados ao longo dos principais vasos sanguíneos debaixo do músculo esternocleidomastóideo em cada lado do pescoço, particularmente o nó da veia jugular interna, com o ângulo da mandíbula. O risco de se espalhar para outras partes do corpo através da corrente sanguínea está intimamente relacionado com a ocorrência do cancer nos gânglios linfáticos no pescoço, quantos nós estão envolvidos e sua localização no pescoço. O risco é mais elevado se o cancro é nos gânglios linfáticos da parte inferior do pescoço, por estarem localizados na parte superior do pescoço ${ }^{8}$.

Segundo Montoro et al. ${ }^{9}$, o comportamento biológico do carcinoma espinocelular - CEC de cavidade oral é incerto, pois há um grande número de tumores que em estádio inicial mostram um comportamento biológico agressivo com metástase regional precoce e morte. Ao contrário, tumores em estádio avançado podem lentamente metastizar-se e esses pacientes, depois do tratamento, obtêm longos períodos livres de doença. O estudo ainda concluiu que a metástase cervical foi o único fator relacionado com o tumor, que mostrou diminuir a sobrevida na sua amostra, confirmando as informações da maioria dos estudos publicados na literatura.

As consequências desse tipo de neoplasia podem afetar profundamente a qualidade de vida - QV das pessoas acometidas, pois o tratamento é agressivo (mutilador) mesmo anos após o tratamento, interferindo nas atividades da vida diária, no que se refere à funcionalidade e à autoimagem ${ }^{10}$.

Tais alterações podem levar a diversas modificações funcionais no organismo do indivíduo, favorecendo adaptações corporais como posturas compensatórias álgicas. Sendo assim, essas modificações podem ocasionar danos provisórios ou permanentes, levando mudanças biomecânicas com agravamento dos sintomas dolorosos e expansão do quadro álgico não tumoral.

Portanto, o propósito deste estudo foi conhecer as alterações funcionais em pacientes com câncer de cabeça e pescoço e a atuação da fisioterapia frente a essas disfunções.

\section{Desenvolvimento}

\subsection{Metodologia}

Foi realizado um estudo de revisão de literatura, com pesquisa dos artigos nas bases de dados eletrônicas, nacionais e internacionais: Medline, Lilacs e Ibecse pelas bibliotecas online Bireme e SciELO. Foram utilizados os seguintes descritores na estratégia de busca pelos artigos: "câncer de cabeça, câncer de pescoço, câncer de boca, associando com a palavra fisioterapia" e "head cancer, neck cancer e physiotherapy". Para conferir maior especificidade à busca, foi utilizado o operador boleano "AND", que permite selecionar artigos que contenham ambos os descritores. Foram incluídos estudos publicados no idioma inglês, português e espanhol, sem restrição quanto ao tipo de estudo e ao período de publicação e que abordassem aspecto clínico do câncer de cabeça e pescoço, prognóstico e reabilitação fisioterapêutica. Após a seleção dos artigos, realizou-se a extração das informações referentes as alterações funcionais em pacientes com câncer de cabeça e pescoço e a atuação da fisioterapia frente a essas disfunções.

\subsection{Discussão}

Os achados do estudo foram organizados em complicações pós-operatórias e as alterações funcionais e a atuação da fisioterapia no câncer de cabeça e pescoço, incluindo o câncer de boca.

Em vários estudos foram encontrados como métodos terapêuticos utilizados nos tumores de cabeça e pescoço a cirurgia, a radioterapia, e a quimioterapia. A complexidade do tratamento e da reabilitação dos pacientes com tumores de cabeça e pescoço exige uma equipe multidisciplinar altamente especializada. Um dos profissionais que trabalha de forma direta com o paciente oncológico é o fisioterapeuta, durante sua reabilitação e também no tratamento paliativo.

\subsubsection{Complicações pós-operatórias e alterações funcionais do câncer de cabeça e pescoço}

De acordo com a literatura, pode-se evidenciar diversas complicações entre elas as mais comumente relatadas são dor e inabilidade funcional no ombro abrangendo diversos sintomas, tais como: limitação na amplitude de movimento; alteração e/ou perda de força na musculatura do membro superior e pescoço homolateral à cirurgia, podendo ocasionar depressão do ombro com deslocamento anterior, dependendo das estruturas removidas e da quantidade de fibrose desenvolvida pela cirurgia e pela radioterapia pós-operatória e, ainda, impacto psicossocial ${ }^{11}$.

Os procedimentos cirúrgicos são bastante invasivos e mutiladores, gerando mutilações temporárias ou definitivas para o paciente. No que diz respeito às alterações fisiológicas advindas da cirurgia, principalmente em casos de câncer de laringe, tem-se a possibilidade do uso de traqueostomia que, se por um lado viabiliza a função respiratória, por outro pode gerar a afonia. $\mathrm{O}$ uso de sonda nasogástrica, associada ou não ao traqueostoma, possibilitará a nutrição desse paciente, mas também pode causar deficiências na deglutição e mastigação, no olfato e no controle de secreções, além de restrição dos movimentos do ombro e do pescoço ${ }^{12,13}$. 
Segundo o Instituto Nacional do Câncer ${ }^{14}$, após uma laringectomia ou outra cirurgia na garganta, pode haver dormência no pescoço e na garganta porque os nervos foram cortados. Se os gânglios linfáticos no pescoço forem removidos, tanto o ombro quanto o pescoço podem tornar-se fracos e rígidos.

Como resultado das intervenções cirúrgicas ou decorrente de sequela de expansão tumoral, os pacientes portadores de câncer de cabeça e pescoço também podem apresentar como complicações o linfedema ${ }^{7,15}$.

O linfedema representa uma falha na drenagem linfática de determinada região da pele, levando ao acúmulo de líquido intersticial. Trata-se de uma condição que causa sofrimento, desconforto permanente, dor e prejuízos funcionais, levando a problemas músculo-esqueléticos secundários e distúrbios psicológicos causados pela desfiguração. As alterações funcionais causadas pelo linfedema facial provocam prejuízos na fala e deglutição e o comprometimento da cicatrização do local operado. Além disso, as deformidades de cabeça e pescoço promovem alterações estéticas que diminuem a autoestima e a qualidade de vida de seus portadores $^{16,17}$.

Outro exemplo é o esvaziamento cervical radical, que ocasiona sequela estética e funcional importante em praticamente todos os pacientes submetidos a esta operação, levando a uma síndrome dolorosa e a perda funcional do membro superior ipsilateral ao procedimento, sendo esta uma complicação importante para o fisioterapeuta. $\mathrm{Na}$ tentativa de diminuir a morbidade deste procedimento, algumas modificações têm sido propostas, as quais incluem preservar as estruturas não linfáticas, como veia jugular interna, o músculo esternocleidomastóideo e o nervo acessório ${ }^{11}$.

Sheikh et al. ${ }^{18}$ observaram que os pacientes submetidos a um procedimento de esvaziamento cervical seletivo ou um esvaziamento cervical radical modificado apresentaram significativamente melhor função do ombro do que os pacientes que haviam sido submetidos esvaziamentos radicais. Além disso, o aumento da idade e um histórico de comorbidades foram associados com pior função do ombro, e fisioterapia pós-operatória foi associada a melhor função do ombro.

Diversas alterações funcionais são causadas em região de ombro pelos esvaziamentos cervicais, como dor que é alegada por ser consequência da disfunção do nervo acessório devido à incapacidade do músculo trapézio de estabilizar a escapula, levando a atrofia desse músculo com consequente queda do ombro, dor e incapacidade de abdução do braço em posição vertical. Isso pode impactar diretamente na qualidade de vida destes pacientes, uma vez que a função de ombro é fundamental na realização de diversas atividades de vida diária. Embora a disfunção do nervo tenha uma relação significante com a dor em ombro, um estudo demonstrou que apenas $51 \%$ dos pacientes com dor tinham disfunção do nervo acessório $^{19}$.
Em geral, há consenso entre os estudos de que os esvaziamentos cervicais mais radicais causam maior disfunção em ombro, e tem sido demonstrado que a disfunção de ombro é o principal problema em longo prazo para estes pacientes ${ }^{7}$.

O nervo frênico também pode ser seccionado ou tracionado, causando elevação da cúpula diafragmática, com consequente desconforto respiratório. A lesão do ramo mandibular do facial quando da dissecção do triangulo submandibular e da elevação dos retalhos cutâneos leva a paralisia da musculatura labial correspondente. $\mathrm{Na}$ dissecção do pescoço, as lesões ao nervo lingual ou hipoglosso, embora raras, aumentam a dificuldade para engolir e falar ${ }^{7}$.

As alterações físicas e funcionais decorrentes do câncer de cabeça e pescoço podem levar o paciente ao isolamento. Tais complicações podem ser classificadas em anatômicas, fisiológicas e técnicas. As anatômicas ocorrem devido a um acidente durante o ato operatório, consistindo de lesões a nervos, vasos ou do ducto torácico durante o esvaziamento cervical ou outros procedimentos na região; as fisiológicas ocorrem como interferência no suprimento de drenagem linfática ou sanguínea e são sequelas praticamente inevitáveis do tratamento, como por exemplo, o edema cerebral, de língua, laringe e face, após o esvaziamento cervical, principalmente quando bilateral; e as técnicas que decorrem de um preparo ineficaz de um procedimento ablativo ou reconstrutivo ${ }^{7,20}$.

Verificaram-se também complicações incomuns, tais como: neuropatia óptica isquêmica anterior em paciente com fatores de risco pré-existentes fratura de clavícula, devido às alterações no movimento de giro do ombro que distorce as forças musculares atuantes sobre a clavícula; tromboembolismo pulmonar em pacientes com doença cardíaca predisponente; paralisia transitória do diafragma; disfagia; fraqueza no lábio inferior após parotidectomias. Em casos de terapia combinada (cirurgia, quimioterapia e radioterapia), pode ocorrer trauma no nervo auriculotemporal, ramo do nervo mandibular, ocasionando, assim, a síndrome auriculotemporal, cujos sintomas clássicos são sudorese gustativa, hiperemia e, raramente dor ${ }^{11}$.

Ao nivel da cabeça e pescoço, altas doses de radiação podem causar hipóxia, redução de suprimento sanguineo, necrose e consequentemente infecção. Da mesma forma, a pele encontrada no campo irradiado poderá sofrer alterações como descamação, formação de bolhas, eritema, necrose, como tambem dor e ardencia nos casos mais severos ${ }^{21}$.

Além disso, o tratamento de radioterapia nesse tipo de câncer pode afetar a articulação temporomandibular e músculos mastigatórios, tendo como consequência o trismo ou limitação da abertura bucal ${ }^{1}$.

Foram encontrados também, alterações como sindrome reto-esfenoidal, provocando sintomas como ofitalmoplegia unilateral, ptose palpebral, dor e trigeminalmias, associados à paralisia unilateral dos músculos da mastigação, sindrome do espaço retroparotídeo,que resulta em dificuldade de 
deglutição, diminuição da gustação e salivação, assim como disfonia associada à paralisia do músculo trapezio e esternocleideomastoide, musculatura unilateral da lingua, palato mole. Tais alterações foram associadas ao câncer em nasofaringe ${ }^{7}$.

Não foram evidenciadas complicações cirúrgicas significativamente diferentes entre pacientes que se submeteram primeiramente à quimioterapia, em relação aos que se submeteram primeiramente à cirurgia. A dor é uma complicação comum, podendo ter diversas origens, ocorrendo em 35\% dos casos de recorrência do câncer, 30\% de sequelas do tratamento, $25 \%$ de causas múltiplas, e $10 \%$ de causas não relacionadas. Portanto, tais pacientes apresentam frequentemente limitações funcionais, resultantes da fadiga, descondicionamento, dor ou metástases musculoesqueléticas e nervosas ${ }^{11}$.

\subsubsection{A atuação da fisioterapia}

A neoplasia de cabeça e pescoço cria problemas estéticos e clínicos se a patologia não for tratada e controlada adequadamente. A complexidade que existe entre face, cavidade oral, nasal, voz e passagem do ar, na aparência pessoal, na ingestão alimentar e no conforto, fazem com que o planejamento terapêutico seja bastante complicado ${ }^{20}$.

Além disso, a literatura aponta para o uso de cinesioterapia, drenagem linfática manual, exercícios respiratórios entre outros como recursos, com o propósito de restaurar ou melhorar as alterações funcionais decorrentes da cirurgia e radioterapia $^{15,17}$.

A fisioterapia deve ser realizada para impedir a perda da funcionalidade, assim como minimizar a dor oncológica, fazendo com que os pacientes tenham diminuição das deformidades, motivando-os assim a continuar o tratamento fisioterapêutico ${ }^{22}$.

Dessa forma, um programa de fisioterapia bem estruturado durante a quimioterapia e radioterapia e no pós-operatório pode desempenhar um papel importante na melhoria da qualidade de vida em pacientes com câncer de cabeça e pescoço, evitando complicações como mucosite oral, diminuição da amplitude de movimento cervical e fadiga relacionada ao câncer que leva à diminuição da capacidade funcional ${ }^{23}$.

Comumente, na presença de dor oncológica, os pacientes reduzem a sua movimentação e sua atividade física como um todo, ocasionando um comprometimento gradual do condicionamento físico, da força muscular, da flexibilidade e da capacidade aeróbica, fatores esses que com frequência levam o paciente à chamada síndrome da imobilização, a qual compromete a coordenação motora, reduz a amplitude de movimento articular e acarreta retrações tendíneas ${ }^{11}$.

Neste contexto, a cinesioterapia é fundamental em todo o processo de reabilitação, podendo ser iniciada com a movimentação passiva no pós-operatório imediato em torno do $3^{\circ}$ dia e a movimentação ativa e exercícios de fortalecimento da musculatura podem ser iniciados assim que os drenos forem retirados. A cinesioterapia nesta etapa se torna um recurso de grande valia, visto que auxilia na restauração e na melhora do desempenho funcional dos segmentos acometidos, desenvolvendo propriocepção, o movimento, a força e o trofismo muscular, prevenindo a imobilidade no leito e devolvendo a amplitude de movimento articular ${ }^{11}$.

As técnicas de terapia manuais podem ser utilizadas como um recurso para a complementação do alívio da dor. A eletroterapia através da estimulação elétrica transcutânea TENS é comumente utilizada para analgesia, como também a termoterapia, através do calor superficial, é usada para reduzir o desconforto e promover o relaxamento muscular através da interferência no ciclo dor-espasmo-dor. Não foram encontrados estudos que comprovem a eficácia da crioterapia para a dor oncológica, mas a sua aplicação pode ser útil para as dores musculoesqueléticas, através de bolsas ou imersão em água gelada, duas a três vezes ao dia, durante 15 a 20 minutos $^{11,23}$.

Segundo McNeely et al. ${ }^{24}$, o treinamento progressivo de exercícios de resistência - TPER reduziu significativamente a dor no ombro e incapacidade e melhora a força muscular do membro superior e resistência em cabeça e pescoço de sobreviventes que tinham disfunção do ombro por causa de lesão do nervo acessório espinhal. Os médicos devem considerar a inclusão de TPER na reabilitação de cabeça póscirúrgico em sobreviventes de câncer no pescoço. A melhoria na dor foi associada ao aumento da força do membro superior e resistência. Os resultados foram consistentes com a hipótese de que a redução da dor pode ser mediada por melhorias na força muscular e resistência. Conforme os autores mencionados, acredita-se que a dor é secundário para a atrofia do músculo do trapézio, o que leva à deslocação descendente e lateral da escápula e inclinação do ombro. O aumento da força dos músculos da cintura escapular pode aliviar a dor, melhorar o posicionamento da escápula e, assim, a mecânica do complexo do ombro.

Além disso, em caso de linfedema, recomenda-se a drenagem linfática manual, uma vez que esta técnica tem se mostrado eficaz na redução do linfedema facial após cirurgia oncológica de cabeça e pescoço, especialmente no período de internação. Apesar da obtenção de bons resultados, o número de sessões variam entre os estudos, bem como o protocolo de realização das sessões, sendo necessárias mais pesquisas para adequações e reprodução da técnica ${ }^{15,25-27}$.

Apesar da dificuldade em encontrar estudos abordando o tratamento fisioterapêutico, a fisioterapia aplicada à oncologia surge como um meio de preservar, manter e restaurar a integridade cinético-funcional dos órgãos e sistemas do paciente oncológico, bem como de prevenir os distúrbios causados pelo tratamento da doença. No entanto, mais pesquisas devem ser realizadas sobre a atuação da fisioterapia no tratamento de pacientes com câncer de cabeça e pescoço. 


\section{Conclusão}

As intervenções cirúrgicas em pacientes com câncer de cabeça e pescoço podem apresentar complicações e alterações funcionais decorrentes da cirurgia, devido à agressão ou adaptações após a retirada dos tumores. Entre elas, estão a alterações funcionais na região do ombro como dor, limitação do movimento, rigidez articular e musculatura mais frágil com perda de força muscular nos membros superiores e pescoço homolateral à cirurgia, podendo levar a depressão com deslocamento anterior e linfedema.

Assim sendo, os recursos fisioterapêuticos como eletroterapia e termoterapia e exercícios terapêuticos (cinesioterapia) podem auxiliar na redução da dor, melhorar a qualidade de vida, minimizar o desconforto, bem como aumentar as funções, como a do braço e ombro.

\section{Referencia}

1. Ribeiro SIM. Alterações na cavidade oral provocadas pelo tratamento de radioterapia em pacientes com cancro de cabeça e pescoço. Dissertação [Mestrado em Ciências da Saúde] - Universidade Fernando Pessoa; 2012.

2. Gallo CVM, Gerra MR, Mendonça GAS. Risco de câncer no Brasil: tendências e estudos epidemiológicos mais recentes. Rev Bras Cancerol 2005;51(3):227-34.

3. Dobrossy L. Epidemiology of head and neck cancer: magnitude ofthe problem. Cancer Metastasis Rev 2005;24:917.

4. Cardoso JP, Pinheiro SMS, Prado FO. Conhecimentos e diagnóstico em câncer bucal entre profissionais de odontologia de Jequié, Bahia. Rev Bras Cancerol 2010;6(2):195-205.

5. Alvarenga LM, Ruiz MT, Bertelli ECP, Ruback MJC, Maniglia JV, Goloni-Bertollo EM. Avaliação epidemiológica de pacientes com câncer de cabeça e pescoço em um hospital universitário do noroeste do estado de São Paulo. Rev Bras Otorrinol 2008;74(1):68-73.

6. Santos VCB, Assis AMA, Ferreira SMS, Dias EP. Câncer de boca: análise do tempo decorrido da detecção ao início do tratamento em centro de Oncologia de Maceió. Rev Bras Odontol 2012;69(2):159-64.

7. Kowalski LP, Magrin J. Complicações das cirurgias por câncer de boca e de orofaringe. Rev Bras Cir Cabeça Pescoço 2003;31(2):45-8.

8. Head and neck cancer treatment. Radiological Society of North America (RSNA) 2014. [acesso em 28 jun 2015]. Disponível em http://www.radiologyinfo.org/en/pdf/hdneck. pdf

9. Montoro JRMC, Hicz HA, Souza L, Livingstone D, Melo $\mathrm{DH}$, Tiveron RC, et al. Fatores prognósticos no carcinoma espinocelular de cavidade oral. Rev Bras Otorrinol 2008;74(6):861-6.

10. Almeida AF, Alves RC, Felix JD, Castro DS, Zandonade E, Rocha RM. Qualidade de vida das pessoas acometidas por câncer no trato aerodigestivo superior em um Hospital Universitário. Rev Bras Cancerol 2013;59(2):229-37.

11. Mozzini CB, Mozzini AR, Schuster RC. O esvaziamento cervical e o papel da fisioterapia na sua reabilitação. Rev Bras Cancerol 2007;53(1):55-61.

12. Leitão BFB, Duarte IV, Bettega PB. Pacientes com câncer de cavidade bucal submetidos à cirurgia: representações sociais acerca do adoecimento e tratamento. Rev SBPH 2013;16(1):113-40.

13. Hannickel S, Zago MMF, Barbeira CBS, Sawada NO. O comportamento dos laringectomizados frente à imagem corporal. Rev Bras Cancerol 2002,48(3):333-9.

14. Instituto Nacional de Câncer - INCA. Fisioterapia. 19962014. 2014. [acesso em 28 jun 2015]. Disponível em http:// www1.inca.gov.br/conteudo_view.asp? ID $=682$

15. Arieiro EG, Machado KS, Lima VP, Tacani RE, Diz AM. A eficácia da drenagem linfática manual no pós-operatório de câncer de cabeça e pescoço. Rev Bras Cir Cabeça Pescoço 2007;36(1):43-6.

16. Mortimer PS. Therapy approaches for lymphedema. Angiology 1997;48(1):87-91.

17. Silva PCF, Araki LT, Agata AC, Becker EF. Fisioterapia respiratória como fator potencializador na recuperaçäo de p.o. De cirurgias de cabeça e pescoço. Fisioter Mov 1999;12(2):15-22.

18. Sheikh A, Shallwani H, Ghaffar S. Postoperative shoulder function after different types of neck dissection in head and neck cancer. Ear Nose Throat J 2014;93(4/5):E21-6.

19. Carvalho APV. Exercícios para disfunção de ombro em pacientes tratados com câncer de cabeça e pescoço: revisão sistemática e metanálises. Dissertação [Mestrado em Ciências] - Universidade Federal de São Paulo; 2011

20. Borges DA visão do enfermeiro frente a alteração da imagem corporal do paciente com câncer de cabeça e pescoço. Porto Alegre: Universidade Federal do Rio Grande do Sul; 2010.

21. Salazar M, Victorino FR, Paranhos LR, Ricci ID, Gaeti WP, Caçador NP. Efeitos e tratamento da radioterapia de cabeça e pescoço de interesse ao cirurgião dentista Revisão da literatura. Rev Odonto 2008;16(31):62-8.

22. Sampaio LR, Moura CV, Resende MA. Recursos fisioterapêuticos no controle da dor oncológica: revisão de literatura. Rev Bras Cancerol 2005;51(4):339-46.

23. Samuel SR, Maiya AG, Vidyasagar MS. Quality of life in head and neck cancer patients: a physiotherapist's perspective. Indian J Cancer 2014;51:86.

24. McNeely ML, Parliament MB, Seikaly H, Jha N, Magee DJ, Haykowsky MJ, et al. Effect of exercise on upper extremity pain and dysfunction in head and neck cancer survivors. Cancer 2008;113(1):214-22.

25. Piso DU, Eckardt A, Liebermann A, Gutenbrunner C, Schäfer P, Gehrke A. Early rehabilitation of head-neck edema after curative surgery for orofacial tumors. Am J Phys Med Rehabil 2001;80(4):261-9.

26. Cobo PC, Marto Diaz PL, Ruiz Molina D, Vaquerizo Garcia E, Suárez Vázquez A, Fernadez Vega A. Drenaje linfático manual em el linfedema facial. Rehabilitación 2003;37(5):79-81.

27. Tacani PM, Franceschini JP, Tacani RE, Machado AF, Montezello D, Góes JC, Marx A. Retrospective study of the physical therapy modalities applied in head and neck lymphedema treatment. Head Neck 2016;38(2):301-8. doi: 10.1002/hed.23899. 